\title{
Arterial spin labeling in the grading of brain gliomas: could it help?
}

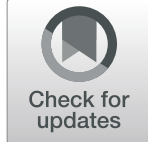

Ahmed A. ElBeheiry ${ }^{1 *}$ D, Doaa M. Emara', Amany Abdel-Bary Abdel-Latif ${ }^{2}$, Mohamed Abbas ${ }^{3}$ and Amal S. Ismail ${ }^{1}$

\begin{abstract}
Background: Gliomas are characterized by high morbidity and mortality with low cure and high recurrence rates, which depends to a great degree on the angiogenesis of the tumor. Assessment of such angiogenesis by perfusion techniques is of utmost importance for the preoperative grading of gliomas. The purpose of this study was to assess the role of arterial spin labeling (ASL) perfusion as a non-contrast MRI technique in the grading of brain gliomas, in correlation with the dynamic susceptibility contrast perfusion imaging (DSC-PI). The study was carried out on 35 patients admitted to the Neurosurgery Department with MRI features of gliomas and sent for further perfusion imaging. Non-contrast ASL followed by DSC-PI was done for all cases. The final diagnosis of the cases was established by histopathology.

Results: Fourteen patients (14/35) had low-grade gliomas while twenty-one (21/35) had high-grade gliomas. In low-grade gliomas, four cases out of 14 were falsely graded as high-grade tumors showing hyperperfusion on ASL, three of which showed DSC-PI hypoperfusion. In high-grade gliomas, two cases out of 21 were interpreted as an indeterminate grade by ASL showing isoperfusion, however showed hyperperfusion on DSC-PI. ROC curve analysis showed ASL-derived rCBF > 2.08 to have $80.95 \%$ sensitivity, $85.71 \%$ specificity, and overall accuracy of $82.86 \%$ compared to $100 \%$ sensitivity, specificity, and accuracy of DSC-PI-derived rCBV and rCBF of $>1.1$ and $>0.9$, respectively. A significant positive correlation was noted between ASL and DSC-PI with correlation coefficient reaching $r=0.80$ between ASL-rCBF and DSC-rCBF $(p<0.01)$ and $r=0.68$ between ASL and DSC-rCBV $(p<0.01)$.

Conclusions: ASL is a relatively recent non-contrast perfusion technique that obtains results which are in fair agreement with the more established DSC perfusion imaging making it an alternative method for preoperative assessment of perfusion of gliomas, especially for patients with contraindications to contrast agents.
\end{abstract}

Keywords: Arterial spin labeling, Dynamic susceptibility contrast perfusion imaging, Gliomas

\section{Background}

Brain tumors are considered one of the most important and frequent difficulties neuroradiologists are facing in their daily routine work. Malignant brain tumors are characterized by abnormal proliferation of tumor cells invading the normal brain with consequent neurological disorders and affection of normal brain functions [1].

Brain tumors arising from glial cells are the most common primary intra-axial cerebral tumors. WHO grading

\footnotetext{
* Correspondence: elbeheiryahmed@gmail.com

'Department of Radiodiagnosis, Faculty of Medicine, University of Alexandria, Alexandria, Egypt

Full list of author information is available at the end of the article
}

of glioma is of value in patients' management and prognosis, where grades I and II are low grade while grades III and IV are more aggressive high-grade gliomas. MRI with novel techniques plays an important role not only in diagnosis but also in differentiating and grading of brain tumors as well as in follow-up after treatment [2].

Contrast-enhanced conventional MR imaging is mostly sufficient for intracranial tumor diagnosis. But there are some limitations, like the non-specificity of contrast enhancement. Enhancement after contrast agent reflects blood-brain barrier disruption rather than a true assessment of tumor vascularity. Especially, the differential diagnosis between high- and low-grade tumors is challenged 
by using only conventional contrast-enhanced cranial MRI [3].

Recent advances in dynamic MR imaging have enabled the assessment of tumor vascularity quantitatively. Among various functional imaging techniques, perfusion MR imaging is particularly sensitive in demonstrating microvasculature and tumor neovascularization [4].

Perfusion MR imaging methods exploit signal changes that accompany the passage of tracer through the cerebrovascular system. The tracer can be endogenous (arterial water) or exogenous (deuterium oxide, gadopentetate dimeglumine). One of the exogenous tracer methods of perfusion imaging is dynamic susceptibility contrast perfusion imaging (DSC-PI). DSC-MRI is based on T2weighted imaging and requires a faster dynamic echoplanar imaging scan with parallel imaging to cover the whole brain. In DSC-PI, the rapid loss of MR signal on T2*-weighted images is measured and then used to calculate the change in concentration of contrast material for each individual voxel [5].

Arterial spin labeling (ASL) is one of the recent, advanced, safe, non-invasive, and non-contrast MRI perfusion techniques by which cerebral blood flow (CBF) can be measured quantitatively and qualitatively using arterial blood water as a freely diffusible endogenous tracer [6]. ASL generates an image by magnetically "labeling" water molecules as an endogenous tracer as they travel to an organ of interest. Selective radiofrequency (RF) irradiation inverts the magnetization of the arterial blood water in the region or plane to which it is applied, usually in the neck for brain perfusion, and a downstream measurement is taken as labeled spins exchange into the tissue of interest. In most ASL methods, the resulting images are compared to control images in which no inversion pulse is applied. The difference reveals the perfusion, indirectly related to the quantification of cerebral blood flow (CBF) in well-characterized units of milliliters of blood per $100 \mathrm{~g}$ of tissue per minute [6].

The aim of this study was to assess the added value of ASL as a non-contrast MRI technique in the grading of brain gliomas and detect its efficacy, sensitivity, and specificity in correlation with the dynamic susceptibility contrast perfusion imaging (DSC-PI).

\section{Methods}

Approval for this cross-sectional prospective study was obtained from the local human research ethics committee. All study procedures were carried out in accordance with the Declaration of Helsinki regarding research involving human subjects [7]. Written informed consent was obtained from all patients.

\section{Study population}

From June 2019 to April 2020, patients with MRI features in favor of gliomas were referred to the Radiodiagnosis Department for further perfusion study. Some patients were excluded including patients with contraindication to DSC-PI due to high creatinine level, post management patients for follow-up to assess residual/recurrent tumor tissue to avoid overlap of the post management perfusion effect upon the original tumor, and patients not pathologically proven as gliomas.

\section{MRI imaging protocol}

MRI examinations were performed with 3T MR scanner (Discovery MR750 w 3.0T, General Electric, Milwaukee, USA). MR images were acquired with the following protocols:

a) Pre-contrast series included axial T1-weighted spinecho (repetition time mseclecho time msec, 600\15), axial, sagittal, and coronal T2-weighted turbo spin-echo (4000\100), axial FLAIR (repetition time mseclinversion time msec, $11000 \backslash 140 \backslash 2200)$, diffusion-weighted imaging with diffusion gradient $b$ values of 0 and $1000 \mathrm{~s} \backslash \mathrm{mm}^{2}$, along three orthogonal axes ( $x, y$, and $z$ directions) over ( $\mathrm{TR}=5072$; a number of sections $=16-22$ ).

b) 3D PCASL combining pseudo-continuous arterial spin labeling [PCASL], and three dimensional [3D] fast spin-echo encoding and spiral trajectory acquisition techniques. The imaging parameters were as follows: TR/TE $=4894 / 10.7 \mathrm{~ms}$, inversion time (TI) was $2025.0 \mathrm{~ms}$, flip angle $=40^{\circ}, \mathrm{FOV}=250 \times 250$ $\mathrm{cm}$, matrix $=512 \times 8$, slice thickness $/$ gap $=4 / 0.0$ $\mathrm{mm}$, number of dynamics $=30$. The total acquisition time was $4 \mathrm{~min}$ with the labeling slab thickness positioned at the level of the upper cervical region.

c) Dynamic susceptibility contrast-enhanced (DSC) gradient-echo echo-planar imaging. The imaging parameters were as follows: TR $\backslash \mathrm{TE} ; 2525 \mathrm{~ms} \backslash 22.1$ ms, flip angle $=35^{\circ}, \mathrm{FOV}=240 \times 240 \mathrm{~cm}$, matrix $=$ $96 \times 128$, slice thickness $/$ gap $=6.5 / 0.0 \mathrm{~mm}$. A series of gradient-echo echo-planar images (40 series) were taken immediately before, during, and after bolus injection of the contrast agent. The T2*weighted DSC perfusion MR images were used in the creation of rCBV and rCBF maps and corresponding $\mathrm{T} 2{ }^{*}$-weighted susceptibility signal intensity-time curves. The total acquisition time was 1 min $30 \mathrm{~s}$.

d) Post contrast series (following injection of gadolinium diethylenetriamine penta-acetic acid (GAD-DTPA) in a dose of $0.1 \mathrm{mmol} / \mathrm{kg}$ body weight were obtained in axial, coronal, and sagittal T1weighted spin-echo sequences. 


\section{Data processing}

- The ASL data were performed on a workstation using Functool Brain stat software (ADW4.7 GE workstation) with automated generation of quantitative perfusion and CBF maps from each subject. A region of interest (ROI) of $4-6 \mathrm{~mm}^{2}$ was drawn over the tumor region showing the highest perfusion value in the CBF map, avoiding the regions of vessels, calcification, hemorrhage, cyst, and necrosis. Another ROI was drawn over the contra-lateral normal-appearing white matter. The value of tumor ROI was divided by the value of contralateral normal WM ROI to estimate relative cerebral blood flow (rCBF) values.

- The DSC data were transferred along with the anatomic data to the same workstation (ADW4.7 GE workstation). T2*-weighted signal intensity-time curves were derived on a voxel by voxel basis. A region of interest (ROI) of 4-6 $\mathrm{mm}^{2}$ was drawn over the tumor region showing the highest perfusion value in the rCBF and rCBV maps, avoiding the regions of vessels, calcification, hemorrhage, cyst, and necrosis. Another ROI was drawn over the contralateral normal-appearing white matter. The value of tumor ROI was divided by the value of contralateral normal WM ROI to estimate relative cerebral blood flow $(\mathrm{rCBF})$ and relative cerebral blood volume (rCBV) values.

- Hyperperfused lesions were interpreted to be of high grade, hypoperfused lesions to be of low grade while isoperfusion was interpreted as an indeterminate grade.

\section{The final diagnosis}

The final diagnosis of the examined brain lesions was established by histopathological analysis.

\section{Statistical analysis}

Data were fed to the computer and analyzed using IBM SPSS software package version 20.0. Data were fed to the computer and analyzed using IBM SPSS software package version 20.0. (Armonk, NY: IBM Corp). KolmogorovSmirnov, Shapiro, and D'agstino tests were used to verify the normality of the distribution of variables. Comparisons between groups for categorical variables were assessed using the chi-square test (Fisher or Monte Carlo). The receiver operating characteristic curve (ROC) was used to determine the diagnostic performance of the markers. The area more than $50 \%$ gives an acceptable performance, and the area about $100 \%$ is the best performance for the test. The significance of the obtained results was judged at the 5\% level. Mann-Whitney test was used to compare between two groups for abnormally distributed quantitative variables.

Spearman's rank correlation analysis was performed to calculate the correlation between ASL-rCBF, DSC-rCBF, and $\mathrm{DSC}-\mathrm{rCBV}$ and pathological diagnosis of gliomas grade.

\section{Results}

\section{Demographic data}

The imaging data of 35 cases with gliomas were used in the present study; 17 were males and 18 were females, ranging in age between 6 and 72 years old (mean, 41.31 years). According to age, 4 groups were identified: $0-18$ years old (pediatric group) included 6 patients (17.1\%), 19-45 years old (youth group) included 15 patients (42.9\%), 46-60 years old (middle-aged group) included 5 patients (14.3\%), and more than 60 years old (elderly group) which included 9 patients (25.7\%).

Hypertension was noted in 14 patients ranging in age between 58 and 72 years, while diabetes mellitus was noted in 11 patients, all of which were above 60 years.

The primary clinical manifestations of enrolled patients included headache, nausea, vomiting, dizziness, seizures, and reduced visual acuity.

\section{Pathological data}

Glioma was pathologically confirmed in all 35 patients. These were classified into 14 low-grade gliomas (LGGs) and 21 high-grade gliomas (HGGs) according to the latest WHO classification criteria (2016). LGGs included 3 cases of ganglioglioma, 4 cases of pilocytic astrocytoma, 3 cases of oligodendroglioma, and 4 cases of grade II astrocytoma. HGGs included 3 cases of anaplastic oligodendroglioma, 7 cases of anaplastic astrocytoma, and 11 cases of grade IV glioblastoma multiform.

In the current study, cases were classified into two groups according to the final pathological diagnosis of the tumors whether low grade or high grade.

Group (1): low-grade gliomas included 14 patients (7 males and 7 females) with mean age \pm SD, $35.8 \pm 18.6$ years, median (min.-max.) 36.5 years (11-64 years) Group (2): high-grade gliomas included 21 patients (10 males and 11 females) with mean age $\pm \mathrm{SD}, 45 \pm 20.1$ years, median (min.-max.) 58 years (6-72 years).

No statistically significant difference was noted between the two groups regarding the demographic data including age, sex, hypertension, and diabetes (Table 1).

\section{Conventional MRI data}

Post contrast sequences in group 1 (low-grade gliomas)

Out of 14 patients with low-grade gliomas, six patients (42.8\%) showed variable degrees of post contrast 
Table 1 Demographic data of the studied cases

\begin{tabular}{llll}
\hline Demographic data & $\begin{array}{l}\text { Group 1 } \\
\text { Low grade }(\boldsymbol{n}=\mathbf{1 4})\end{array}$ & $\begin{array}{l}\text { Group 2 } \\
\text { High grade }(\boldsymbol{n}=\mathbf{2 1})\end{array}$ & Test of sig. \\
\hline Sex & $7(50 \%)$ & $10(47.6 \%)$ & $X^{2}=0.019$ \\
$\quad$ Male & $7(50 \%)$ & $11(52.4 \%)$ & 0.890 \\
$\quad$ Female & & & $U=118.0$ \\
Age (years) & $35.8 \pm 18.6$ & $45 \pm 20.1$ & 0.342 \\
$\quad$ Mean \pm SD & $36.5(11-64)$ & $58(6-72)$ & $X^{2}=0.079$ \\
$\quad$ Median (min.-max.) & 6 & 8 & $x^{2}=0.199$ \\
Hypertension & 5 & 6 & 0.778 \\
Diabetes mellitus & 5 & 0.721 \\
\hline
\end{tabular}

$X^{2}$ chi-square test, $U$ Mann-Whitney test, $p p$ value for comparing between the studied groups, *statistically significant at $p \leq 0.05$

enhancement with eight patients showing no definite enhancement.

\section{Post contrast sequences in group 2 (high-grade gliomas)}

Out of 21 patients with high-grade gliomas, five patients $(23.8 \%)$ did not show any post contrast enhancement with the rest of the patients (16 patients) showing variable degrees of enhancement.

Dynamic susceptibility contrast perfusion imaging (DSCPI) data

DSC-PI findings in group 1 (low-grade gliomas)

DSC-PI showed hypoperfusion in 12 out of the 14 patients with LGG with only two cases showing isoperfusion pattern.

- Hypoperfused lesions showed DSC-PI absolute CBF and CBV of mean \pm SD $11.9 \pm 9.1 \mathrm{ml} / 100 \mathrm{gm} / \mathrm{min}$ and $1.5 \pm 0.67 \mathrm{ml} / 100 \mathrm{gm}$, respectively, and $\mathrm{rCBF}$ $(\mathrm{WM})$ and $\mathrm{rCBV}(\mathrm{WM})$ of mean $\pm \mathrm{SD} 0.63 \pm 0.16$ and $0.73 \pm 0.15$, respectively.

- Isoperfused lesions showed DSC-PI absolute CBF and CBV ranging from 30.5 to 33.5 and 1.9 to 2.1, respectively, and $\mathrm{rCBF}$ (WM) and $\mathrm{rCBV}$ (WM) of 0.9 and 1.1 , respectively.

\section{DSC-PI findings in group 2 (high-grade gliomas)}

All patients with HGG showed hyperperfusion on DSC-PI.

- Hyperperfused lesions showed DSC-PI absolute CBF and CBV ranging from 1.3 to 310 with mean \pm SD of $69.5 \pm 82.6 \mathrm{ml} / 100 \mathrm{gm} / \mathrm{min}$ and 0.35 to 13.3 with mean $\pm \mathrm{SD}$ of $6.7 \pm 3.7 \mathrm{ml} / 100 \mathrm{gm}$, respectively, and $\mathrm{rCBF}$ (WM) and $\mathrm{rCBV}$ ranging from 2.1 to 12.1 with mean \pm SD of $4.3 \pm 2.8$ and 1.4 to 5.7 with mean \pm $\mathrm{SD}$ of $3.3 \pm 1.2$, respectively.

Statistically significant difference was noted between patients of the two groups as regards DSC-CBF and
DSC-CBV including their absolute and relative values (Table 2).

\section{Arterial spin labeling perfusion imaging (ASL) data ASL findings in group 1 (low-grade gliomas)}

ASL showed hypoperfusion pattern in 9 out of the 14 patients with LGG, with 4 cases showing hyperperfusion and one showing isoperfusion.

- Hypoperfused lesions showed ASL perfusion absolute CBF ranging from 14 to $17 \mathrm{ml} / 100 \mathrm{gm} / \mathrm{min}$ with $\mathrm{rCBF}$ (WM) ranging from 0.62 to 0.72 .

- The lesions with hyperperfused nature showed absolute CBF ranging from 60 to $78.5 \mathrm{ml} / 100 \mathrm{gm} /$ min with rCBF (WM) ranging from 2.08 to 3.03 .

- The last lesion with isoperfusion showed absolute CBF of $34 \mathrm{ml} / 100 \mathrm{gm} / \mathrm{min}$ and $\mathrm{rCBF}$ of 1.2 .

\section{ASL findings in group 2 (high-grade gliomas)}

Hyperperfusion was noted in 19 out of the 21 patients with HGG with ASL-derived absolute CBF averaging $55.7-305 \mathrm{ml} / 100 \mathrm{gm} / \mathrm{min}$ and $\mathrm{rCBF}$ averaging 4.1-11.3.

Only two patients showed isoperfused pattern on ASL with absolute CBF of $40-40.5 \mathrm{ml} / 100 \mathrm{gm} / \mathrm{min}$ and $\mathrm{rCBF}$ of 1.20-1.25.

Statistically significant difference was noted between patients of the two groups as regards ASL-derived CBF including its absolute and relative values (Table 3).

\section{ASL versus DSC perfusion}

\section{ASL versus DSC in group 1: low-grade gliomas}

Four cases out of 14 were falsely graded as high-grade tumors showing hyperperfusion on ASL, however proved pathologically to be low-grade tumors (three of which were oligodendrogliomas and the last was grade II astrocytoma). One of these lesions was isoperfused on DSC and the last three showed DSC-PI hypoperfusion. None of these 4 cases showed post contrast enhancement on conventional sequences (Figs. 1 and 2). 
Table 2 DSC-PI findings in the studied groups

\begin{tabular}{|c|c|c|c|c|}
\hline & $\begin{array}{l}\text { Group } 1 \\
\text { Low grade }(n=14)\end{array}$ & $\begin{array}{l}\text { Group } 2 \\
\text { High grade }(n=21)\end{array}$ & Test of sig. & $p$ \\
\hline \multicolumn{5}{|l|}{ DSC-CBF (lesion) } \\
\hline Mean \pm SD & $11.9 \pm 9.1$ & $69.5 \pm 82.6$ & $U=42.0^{*}$ & $<0.001^{*}$ \\
\hline Median (min.-max.) & $8.5(4.5-33.5)$ & $36(1.3-310)$ & & \\
\hline \multicolumn{5}{|l|}{ DSC relative CBF (WM) } \\
\hline Mean \pm SD & $0.63 \pm 0.16$ & $4.3 \pm 2.8$ & $U=0.0^{*}$ & $<0.001^{*}$ \\
\hline Median (min.-max.) & $0.58(0.40-0.90)$ & $3(2.1-12.1)$ & & \\
\hline \multicolumn{5}{|l|}{ DSC-CBV (lesion) } \\
\hline Mean \pm SD & $1.5 \pm 0.67$ & $6.7 \pm 3.7$ & $U=31.0$ & $<0.001^{*}$ \\
\hline Median (min.-max.) & $1.3(0.8-3.1)$ & $6.2(0.35-13.3)$ & & \\
\hline \multicolumn{5}{|l|}{ DSC relative CBV (WM) } \\
\hline Mean \pm SD. & $0.73 \pm 0.15$ & $3.3 \pm 1.2$ & $U=0.0^{*}$ & $<0.001^{*}$ \\
\hline Median (min.-max.) & $0.65(0.60-1.1)$ & $3.1(1.4-5.7)$ & & \\
\hline
\end{tabular}

U Mann-Whitney test, $p p$ value for comparing between the studied groups, * statistically significant at $p \leq 0.05$

\section{ASL versus DSC in group 2: high-grade gliomas}

Two cases out of 21 were interpreted as an indeterminate grade by ASL showing isoperfusion, however proved pathologically to be high grade and showed hyperperfusion on DSC-PI (Fig. 3).

ROC curve analysis showed ASL-derived rCBF > 2.08 to have $80.95 \%$ sensitivity and $85.71 \%$ specificity and overall accuracy of $82.86 \%$ compared to $100 \%$ sensitivity, specificity, and accuracy of DSC-PI-derived rCBV and rCBF of $>1.1$ and $>0.9$, respectively, in differentiation between low- and high-grade gliomas (Tables 4 and 5, Fig. 4).

Correlation analysis between ASL and DSC-PI demonstrated that ASL-rCBF had a significantly positive correlation with both DSC-rCBF $(r=0.800, p<0.01)$ and DSC-rCBV $(r=0.689, p<0.01)$, best and significantly detected in cases of high-grade gliomas (Table 6).

\section{Discussion}

Some of the primary brain tumors mainly gliomas are characterized by high morbidity and mortality with low cure and high recurrence rates; this depends to a great degree on the angiogenesis of the tumor beside the location of the lesion and its accessibility to surgery [8].

Conventional MRI sequences are not very sensitive for the detection of tumor angiogenesis and thus grading of lesions. In conventional MRI, there is overlap in findings between low- and high-grade gliomas, with some highgrade tumors showing no enhancement and some lowgrade lesions showing avid post contrast enhancement, as contrast enhancement depends on disturbance of blood-brain barrier, not on tumor vascularization or angiogenesis [9].

This finding was confirmed in the current study as out of 35 cases studied, six out of the 14 low-grade gliomas (42.8\%) showed variable degrees of post contrast enhancement and five out of the 21 high-grade gliomas (23.8\%) did not show post contrast enhancement. Such results confirm that enhancement is not a reliable factor for determining the tumoral grade, similarly with previous results.

In order to answer the question of the title of the study and understand the role of ASL-perfusion in the grading of gliomas, the current study tried to find an

Table 3 ASL findings in the studied groups

\begin{tabular}{llll}
\hline & $\begin{array}{l}\text { Group 1 } \\
\text { Low grade }(\boldsymbol{n}=\mathbf{1 4})\end{array}$ & $\begin{array}{l}\text { Group 2 } \\
\text { High grade }(\boldsymbol{n}=\mathbf{2 1}\end{array}$ & Test of sig. \\
\hline ASL absolute CBF (lesion) & & & $\boldsymbol{p}$ \\
$\quad$ Mean \pm SD. & $32.4 \pm 25.4$ & $91.9 \pm 74$ & $U=47.50^{*}$ \\
$\quad$ Median (min.-max.) & $17(14-78.5)$ & $60(40.3-305)$ & $<0.001^{*}$ \\
ASL relative CBF (WM) & $1.2 \pm 0.91$ & $4.1 \pm 2.6$ & $U=22.0$ \\
$\quad$ Mean \pm SD. & $0.70(0.62-3)$ & $4.1(1.2-11.3)$ & $<0.001^{*}$ \\
Median (min.-max.) & &
\end{tabular}

$U$ Mann-Whitney test, $p p$ value for comparing between the studied groups, *statistically significant at $p \leq 0.05$ 

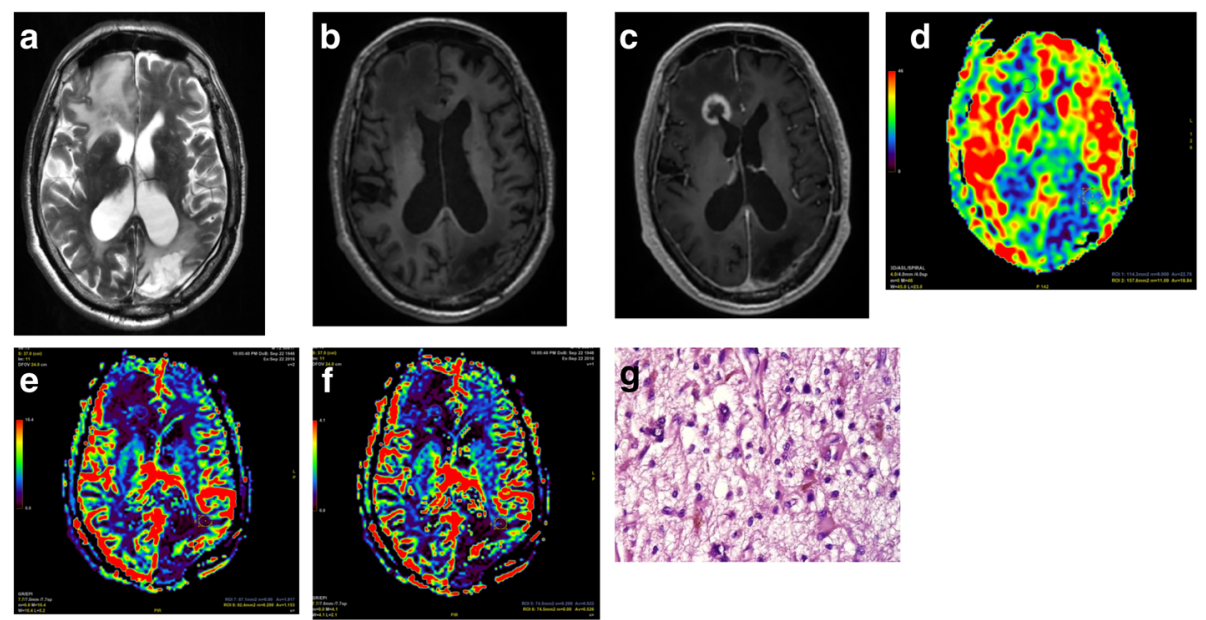

Fig. 1 MR perfusion in a case with the right periventricular low-grade glioma. a Axial T2WI showing right frontal periventricular slightly hypointense lesion surrounded by vasogenic edema. b, c Pre- and post contrast T1WI showing isointense signal with hypointense edema with manifest post contrast enhancement of the periventricular lesion. $\mathbf{d}$ ASL-CBF showing iso to hypoperfusion of the lesion with ASL-rCBF of 0.72. e, $\mathbf{f}$ DSC-CBF and CBV showing hypoperfusion with DSC-rCBF of 0.47 and DSC-rCBV of 0.62. $\mathbf{g}$ Hematoxylin and eosin staining ( $\times 400$ magnification) showing moderate cellularity and moderate nuclear enlargement and pleomorphism with few vessels, pathologically proven low-grade NOS WHO grade II glioma with ASL matching with DSC in showing hypoperfusion of the lesion. ASL arterial spin labeling, CBF cerebral blood flow, DSC dynamic susceptibility contrast, CBV cerebral blood volume, NOS not otherwise specified, $r$ relative, WI weighted imaging

answer to the following questions: Is post contrast DSCperfusion accurate in estimating the grade of gliomas? and Are ASL-perfusion results comparable to the more established post contrast DSC-perfusion in accurate prediction of glioma grading?
Perfusion MRI techniques non-invasively estimate the functional hemodynamic parameters of the brain including tumors angiogenesis. Many different MRI techniques are available including dynamic susceptibility contrast (DSC) as well as dynamic contrast-enhanced (DCE) T1-
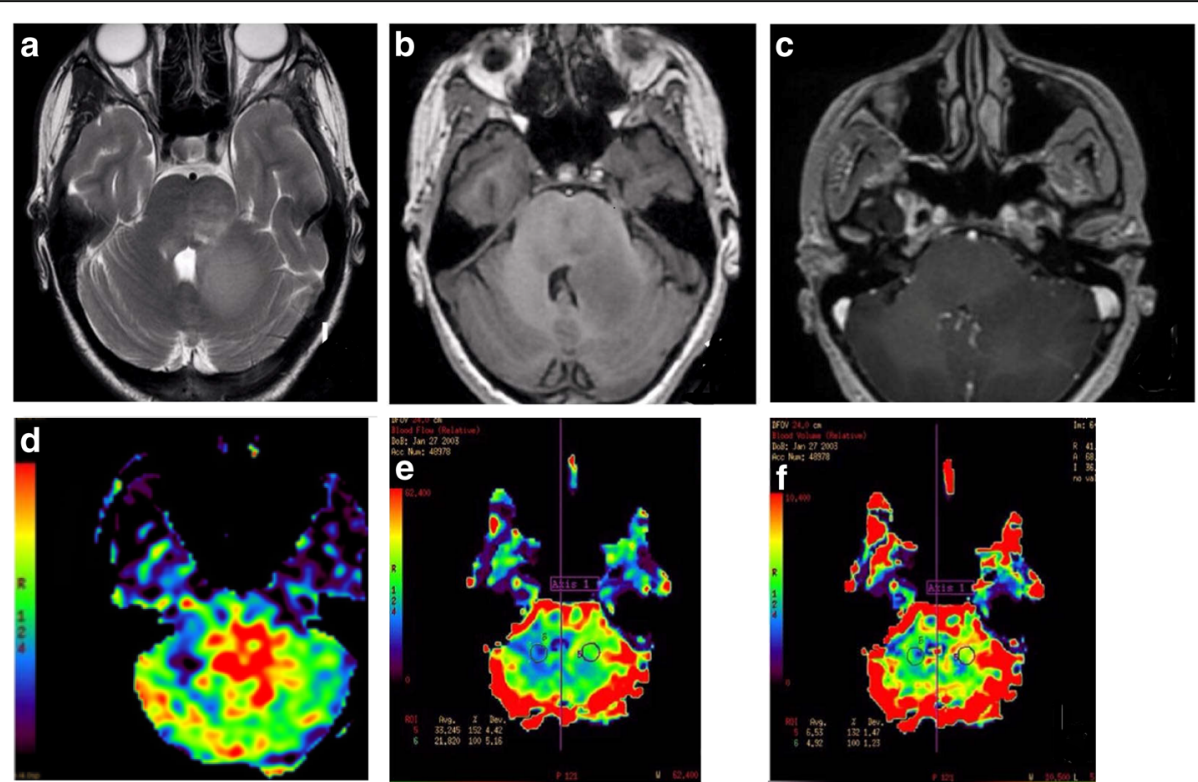

Fig. 2 MR perfusion in a case with the left middle cerebellar peduncle (MCP) low-grade glioma. a Axial T2WI showing left MCP hyperintense lesion. b, c Pre- and post contrast T1WI showing hypointense signal with no post contrast enhancement. $\mathbf{d}$ ASL-CBF showing hyperperfusion of the lesion with ASL-rCBF value of 4.76. e, $\mathbf{f}$ DSC-CBF and CBV showing isoperfusion with a DSC-rCBF value of 0.9 and DSC-rCBV value of 1.1. Pathologically proven low-grade glioma with ASL not matching with DSC, hyper on ASL and iso on DSC. ASL arterial spin labeling, CBF cerebral blood flow, DSC dynamic susceptibility contrast, CBV cerebral blood volume, $r$ relative, WI weighted imaging 

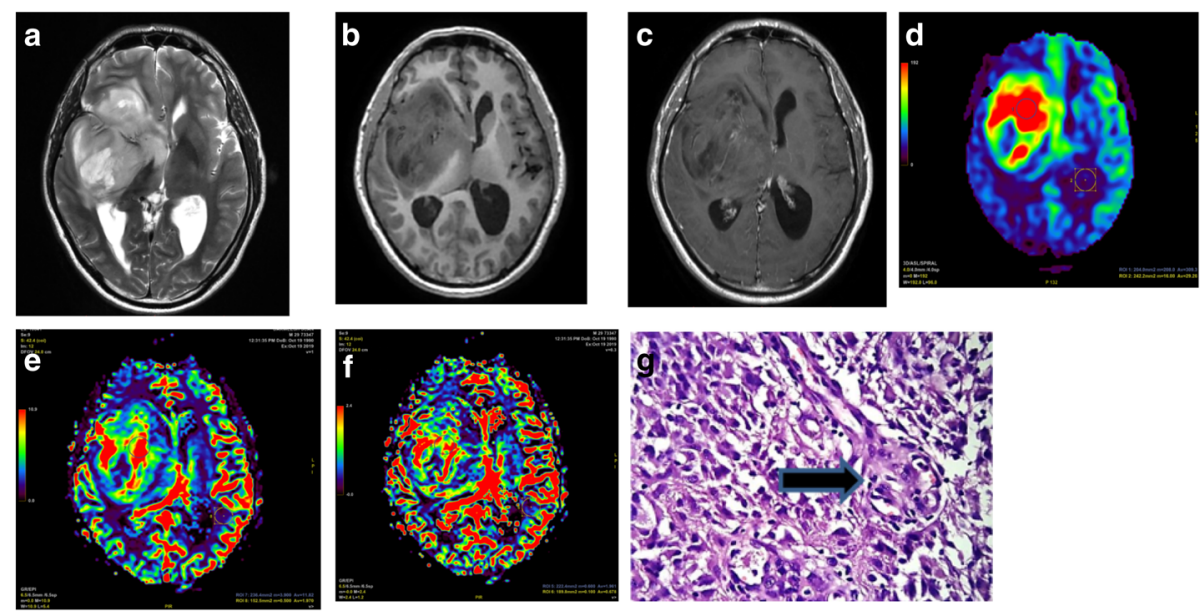

Fig. 3 MR perfusion in a case with the right frontal high-grade glioma. a Axial T2WI showing right frontal hyperintense signal sizable lesion. b, c Pre- and post contrast T1WI showing hypointense signal with patchy faint post contrast enhancement. $\mathbf{d}$ ASL-CBF showing a high perfusion of the lesion with ASL-rCBF value of 8.03. e, $\mathbf{f}$ DSC-CBF and CBV showing a high perfusion with DSC-rCBF value of 4.16 and DSC-rCBV value of 8.16. g Hematoxylin and eosin staining ( $\times 400$ magnification) showing increased cellularity with vascular proliferation (arrows), palisading necrosis and marked cellular atypia, pathologically proven glioblastoma, NOS WHO grade IV with ASL matching with DSC in showing hyperperfusion of the lesion. ASL arterial spin labeling, CBF cerebral blood flow, DSC dynamic susceptibility contrast, CBV cerebral blood volume, NOS not otherwise specified, $r$ relative, WI weighted imaging

weighted perfusion imaging along with non-contrast arterial spin-labeling (ASL) perfusion [3].

Is post contrast DSC-perfusion accurate in estimating the grade of gliomas?

DSC perfusion imaging is the most commonly used modality for perfusion assessment in clinical practice. Previous studies reported that DSC-PI provided useful information about glioma grading [3, 10]. Our findings confirm those of previous studies showing the best positive correlation between the degree of DSC-perfusion parameters and pathologically proven tumor grade.
The rCBV calculated out of DSC-PI is commonly used in evaluating perfusion in brain tumors, but some studies also used rCBF for grading gliomas [10]. According to our results, both $\mathrm{rCBV}$ and $\mathrm{rCBF}$ were significantly different between low- and high-grade gliomas. Our study showed that rCBF higher than 0.9 and rCBV higher than 1.1 showed the highest sensitivity, specificity, and predictive values in the estimation of high-grade gliomas. Such values were less than those produced in the study of Cebeci et al. [11], who found that a cutoff value of 1.80 for rCBV had a higher specificity than the cutoff value of 1.36 for $\mathrm{rCBF}$ for the prediction of high-grade gliomas.

Table 4 Comparison between the two studied groups according to different MRI perfusion parameters

\begin{tabular}{|c|c|c|c|c|}
\hline MR perfusion & Low grade $(n=14)$ & High grade $(n=21)$ & $x^{2}$ & ${ }^{M C} p$ \\
\hline \multicolumn{5}{|l|}{ ASL-CBF } \\
\hline Hypo & $9(64.3 \%)$ & $0(0 \%)$ & 19.057 & $<0.001^{*}$ \\
\hline ISO & $1(7.1 \%)$ & $2(9.5 \%)$ & & \\
\hline Hyper & $4(28.6 \%)$ & 19 (90.5\%) & & \\
\hline \multicolumn{5}{|l|}{ DSC-CBF } \\
\hline Hypo & $12(85.7 \%)$ & $0(0 \%)$ & $39.641^{*}$ & $<0.001^{*}$ \\
\hline ISO & $2(14.3 \%)$ & $0(0 \%)$ & & \\
\hline Hyper & $0(0 \%)$ & $21(100 \%)$ & & \\
\hline \multicolumn{5}{|l|}{ DSC-CBV } \\
\hline Hypo & $12(85.7 \%)$ & $0(0 \%)$ & 37.087 & $<0.001^{\circ}$ \\
\hline ISO & $2(14.3 \%)$ & $1(4.8 \%)$ & & \\
\hline Hyper & $0(0 \%)$ & $20(95.2 \%)$ & & \\
\hline
\end{tabular}

$X^{2}$ chi-square test, MC Monte Carlo, U Mann-Whitney test, $p p$ value for comparing between the studied groups, *statistically significant at $p \leq 0.05$ 
Table 5 Agreement (sensitivity, specificity) for ASL relative CBF (WM), DSC relative CBF (WM), and DSC relative CBV (WM) to differentiate high $(n=21)$ from low $(n=14)$ grade gliomas

\begin{tabular}{llllllllll}
\hline & AUC & $\boldsymbol{p}$ & $\mathbf{9 5 \%}$ C.I & Cutoff & Sensitivity & Specificity & PPV & NPV & Accuracy \\
\hline ASL relative CBF (WM) & $0.925^{*}$ & $<0.001^{*}$ & $0.84-1.01$ & $>\mathbf{2 . 0 8}$ & 80.95 & 85.71 & 89.5 & 75.0 & 82.86 \\
DSC relative CBF (WM) & $1.000^{*}$ & $<0.001^{*}$ & $1.00-1.00$ & $>\mathbf{0 . 9}$ & 100.0 & 100.0 & 100.0 & 100.0 & 100.0 \\
DSC relative CBV (WM) & $1.000^{*}$ & $<0.001^{*}$ & $1.00-1.00$ & $>\mathbf{1 . 1}$ & 100.0 & 100.0 & 100.0 & 100.0 & 100.0
\end{tabular}

AUC area under a curve, $p$ value probability value, $C l$ confidence interval, NPV negative predictive value, PPV positive predictive value, ${ }^{*}$ statistically significant at $p$ $\leq 0.05$

Are ASL-perfusion results comparable to the more established post contrast DSC-perfusion in the prediction of glioma grading?

No need for intravenous contrast agent injection is the major advantage of ASL, which makes ASL easy repeatable. ASL may thus be useful in patients with renal failure, because they may be at risk for contrast-related nephrogenic systemic fibrosis, and in children for whom the intravenous rapid bolus injection of contrast agents may be difficult [12].

According to our results, ASL perfusion parameters, including both absolute and relative cerebral blood flow, could differentiate low- and high-grade tumors. Absolute CBF values obtained with ASL were capable of differentiating tumoral grade, but we think relative values were more reliable.
Because there are individual differences in CBF values, in order to ensure accuracy of the quantitative measurement of $\mathrm{CBF}$, the present study used a ratio of $\mathrm{CBF}$ values of white matter from the contralateral side as quantitative indicators to attain the relative CBF.

Some studies stated that using white matter as a reference region is still questionable due to the long transit time and high water content of the normally appearing white matter in brain tumors, and this may result in underestimation of white matter CBF by ASL [13]. However, in our study as well as Jiang et al. study [14], the white matter was used as a reference region and revealed a highly strong correlation between ASL and DSC.

The vascular pathology of low-grade glioma states that tumor vessels are mainly formed of normal endothelial cells, with cell-to-cell tight junction and relatively intact

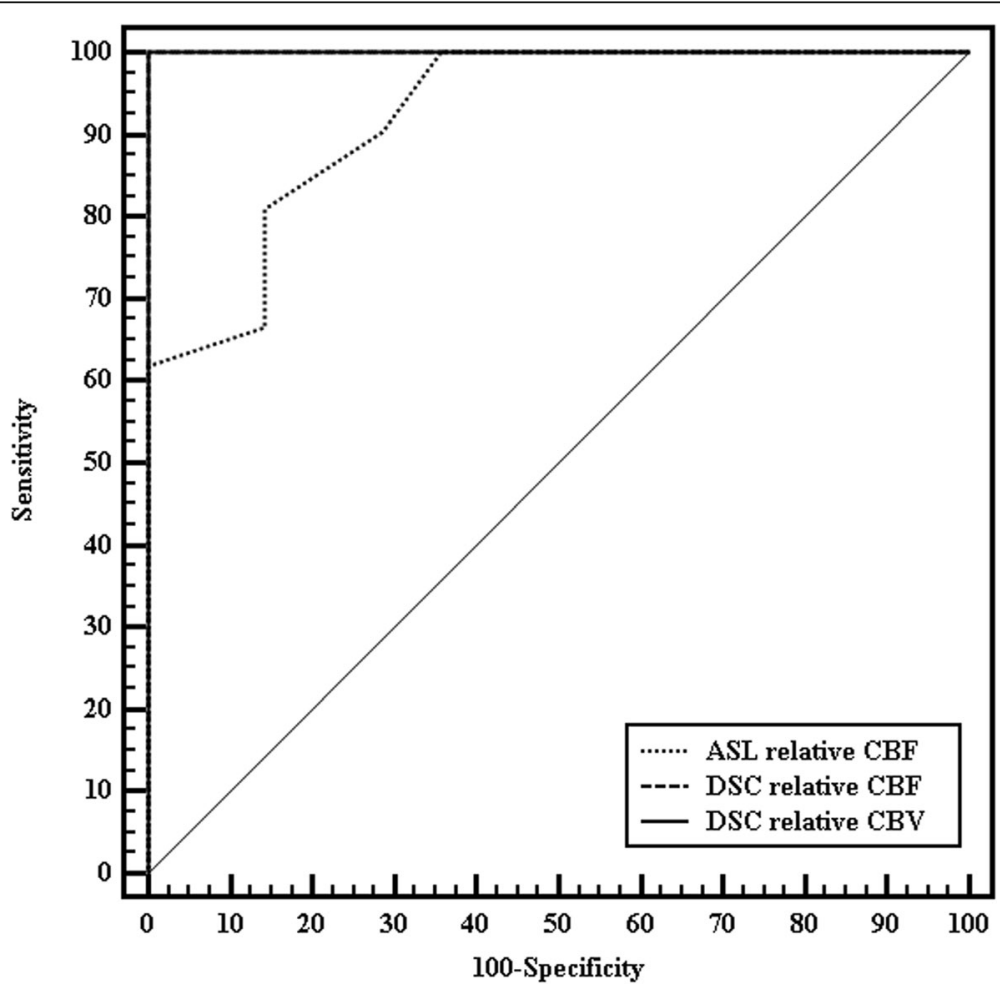

Fig. 4 ROC curve for ASL relative CBF (WM), DSC relative CBF (WM), and DSC relative CBV (WM) to differentiate high $(n=21)$ from low $(n=14)$ grade gliomas 
Table 6 Correlation between ASL rCBF, DSC-PI rCBF, and rCBV in each group (low- and high-grade gliomas) and total sample

\begin{tabular}{|c|c|c|c|c|c|c|}
\hline & \multicolumn{6}{|c|}{ ASL relative CBF (WM) } \\
\hline & \multicolumn{2}{|c|}{ Low $(n=14)$} & \multicolumn{2}{|c|}{ High $(n=21)$} & \multicolumn{2}{|c|}{ Total $(n=35)$} \\
\hline & $r_{\mathrm{s}}$ & $p$ & $r_{\mathrm{s}}$ & $p$ & $r_{\mathrm{s}}$ & $p$ \\
\hline DSC relative CBF (WM) & -0.136 & 0.642 & $0.744^{*}$ & $<0.001^{*}$ & $0.800^{*}$ & $<0.001^{*}$ \\
\hline DSC relative CBV (WM) & 0.022 & 0.939 & 0.245 & 0.285 & $0.689^{*}$ & $<0.001^{*}$ \\
\hline
\end{tabular}

rs Spearman coefficient, ${ }^{*}$ statistically significant at $p \leq 0.05$

blood-brain barrier resulting in decreased blood flow and blood volume parameters in perfusion MRI techniques [3]. Most of the previous studies differentiating high-grade glioma from low-grade glioma by perfusion MRI techniques revealed low perfusion parameters in low-grade glioma patients $[15,16]$.

In our study, 9 out of 14 low-grade glioma patients showed hypoperfusion in ASL-CBF comparable to DSCPI, while four cases showed hyperperfusion on ASL, three of which were hypoperfused on DSC-PI. The last case was isoperfused on ASL and interpreted as an indeterminate grade.

An explanation for such false hyperperfusion in some low-grade gliomas on ASL could be attributed to the nature of these lesions; as three out of the four lesions were proved to be grade II oligodendrogliomas on pathology. Lev et al. [17] investigated glial tumor grade by DSC-PI and found that there were confounding effects of oligodendrogliomas so that two low-grade oligodendrogliomas showed rCBV value higher than the threshold for low-grade lesions. Such finding was confirmed in the current study but only in ASL where $\mathrm{rCBF}$ was higher than the threshold for low-grade oligodendroglioma. Lev et al. [17] found that given the fine capillary network that is typical of even low-grade oligodendrogliomas, it is not surprising that their perfusion pattern should differ from that of low-grade astrocytomas and therefore can confound the reliability of perfusion values in distinguishing high-grade from low-grade untreated glial cell tumors [17].

On the other hand, 19 out of 21 cases with high-grade gliomas in the current study showed hyperperfusion in ASL while all 21 cases showed hyperperfusion in DSC. These results agreed with the previous studies of Cebeci et al. [11], Soni et al. [13], and Jiang et al. [14] who stated that high-grade gliomas consistently show hyperperfusion on ASL perfusion; explained by the vascular histopathological features of such gliomas that have copious neovascularization characterized by disorganized, irregular, and tortuous vessels with arteriovenous shunting resulting in increased perfusion.

Comparing ASL perfusion to DSC-PI has shown ASLderived rCBF $>2.08$ to have $80.95 \%$ sensitivity, $85.71 \%$ specificity, and overall accuracy of $82.86 \%$ compared to
$100 \%$ sensitivity, specificity, and accuracy of DSC-PIderived $\mathrm{rCBV}$ and $\mathrm{rCBF}$ of $>1.1$ and $>0.9$, respectively.

Some discrepancy was noted with the latest study of Hong et al. [18] in which ASL-rCBF had the largest area under the ROC curve (0.836). When the cutoffs for ASL-rCBF, DSC-rCBF, and DSC-rCBV were taken (2.24, 1.85, and 1.68), the sensitivities of HGG diagnosis were $83.2,91.3$, and $91.3 \%$, and the specificities were 77.7 , 63.9 , and $66.7 \%$, respectively.

Such discrepancy in results is likely attributed to the difference in taking the reference values as in their study, the contralateral normal ROI was selected by using the mirror image of the tumor ROI in contrast to our study where the contralateral normal-appearing white matter was taken as reference.

Spearman correlation analysis between the two perfusion techniques demonstrated a strong positive correlation between ASL-derived rCBF and DSC-rCBF $(r$ reaching 0.8 with $p<0.001)$ and less yet still fair correlation with $\mathrm{rCBV}$ ( $r$ reaching 0.68 with $p<0.001$ ).

A strong positive correlation between ASL and DSC has been reported for grading of gliomas in a few recent studies. A nearly similar study by Cebeci et al. [11] on 33 patients (20 high-grade and 13 low-grade gliomas) revealed a significant positive correlation between DSCrCBV and rCBF-ASL $(r=0.81, p<0.001)$. However, the correlation between DSC-rCBF and rCBF-ASL was weaker $(r=0.64, p<0.001)$.

Soni et al. [13] studied 30 cases of brain tumors, eight of which were gliomas and found a high correlation reaching 0.9 between ASL-rCBF and DSC-rCBF when compared to gray matter.

A less but still significant correlation was noted in Hong et al. study [18] on 50 glioma patients, as they found that Spearman correlation analysis demonstrated that ASLrCBF had a significantly positive correlation with DSC$\mathrm{rCBF}(r=0.580, P<0.01)$ and significantly moderate positive correlation with DSC-rCBV $(r=0.431, P<0.01)$.

In general, most of the studies support the opinion that ASL could be a noninvasive alternative to DSC.

However, 3D ASL has a number of deficiencies compared with DSC-PWI. Firstly, 3D ASL has a relatively poor signal-to-noise ratio when compared to DSC. Secondly, 3D ASL cannot measure as many parameters as 
DSC-PWI and has only one CBF parameter, which limits its application. Thirdly, aging and carotid atherosclerosis affect cerebral perfusion [12] and lastly, the longer acquisition time of ASL reaching $4 \mathrm{~min}$ compared to $1 \mathrm{~min}$ and $30 \mathrm{~s}$ of DSC-PI.

\section{Points of strength}

All lesions in the current study had histologically proven diagnoses, and all MR imaging examinations were done at a high magnetic field of $3 \mathrm{~T}$ with a higher signal to noise ratio (SNR), thus leading to decreased motion artifacts compared to $1.5 \mathrm{~T}$. Both perfusion techniques (DSC-PI and ASL) were done in all patients to facilitate comparison data.

\section{Limitations of the study}

The main limitation of the current study was doing singlephase ASL which we think may have resulted in the relatively lower sensitivity and specificity data as compared to the DSC-PI. Still, the ASL technique with multiple repetitions usually causes longer imaging times which should be taken into consideration versus the main advantage of this technique being a non-contrast study.

\section{Conclusion}

Both perfusion MRI techniques, ASL and DSC, were successful in discriminating low- and high-grade gliomas with DSC showing higher sensitivity and specificity. Despite this fact, ASL is still a promising perfusion imaging method having the advantage of being non-invasive. DSC has the advantage of high SNR and lower imaging time. However, a relatively new noninvasive perfusion method, ASL, may obtain results that are in good agreement with DSC perfusion imaging, and it may be a useful alternative method for evaluating the perfusion of glial tumors, especially for patients with contraindications to contrast agents.

\section{Abbreviations \\ LGG: Low-grade glioma; HGG: High-grade glioma; DSC-PI: Dynamic susceptibility contrast perfusion imaging; ASL: Arterial spin labeling; CBF: Cerebral blood flow; CBV: Cerebral blood volume}

\section{Acknowledgements}

Not applicable

\section{Authors' contributions}

$A B$ and $D E$ drafted the manuscript. $A B$ and $A S$ edited the images. $A A$ reviewed the pathology of the lesions. MA performed the surgical interventions for the patients. The authors read and approved the final manuscript.

\section{Funding}

Not applicable

\section{Availability of data and materials}

The datasets used and/or analyzed during the current study are available from the corresponding author on reasonable request.

\section{Ethics approval and consent to participate}

Approval for this cross-sectional prospective study was obtained from the Research Ethics Committee of Alexandria Faculty of Medicine (Ethics committee's reference number: 0304584, IRB No: 00012098, FWA No: 00018699). All study procedures were carried out in accordance with the Declaration of Helsinki regarding research involving human subjects. Written informed consent was obtained from the patients.

\section{Consent for publication}

Written informed consent for publication of this data was given by the patients.

\section{Competing interests}

The authors declare that they have no competing interests.

\section{Author details}

${ }^{1}$ Department of Radiodiagnosis, Faculty of Medicine, University of Alexandria, Alexandria, Egypt. ${ }^{2}$ Department of Pathology, Faculty of Medicine, University of Alexandria, Alexandria, Egypt. ${ }^{3}$ Department of Neurosurgery, Faculty of Medicine, University of Alexandria, Alexandria, Egypt.

Received: 24 August 2020 Accepted: 8 November 2020

Published online: 20 November 2020

\section{References}

1. Akira H, Tomohiro K, Kei N, Ayumi N, Masafumi M, Masaya K, et al (2019) Treatment strategies based on histological targets against invasive and resistant glioblastoma. J Oncol 2019:2964783

2. Belén D, Miguel Á (2020) Advances in the knowledge of the molecular biology of glioblastoma and its impact in patient diagnosis, stratification, and treatment. Adv Sci 7:1902971

3. Zhang J, Heng L, Haipeng T, Sumei W, Yizeng Y, Gang L et al (2017) Clinical applications of contrast-enhanced perfusion MRI techniques in gliomas: recent advances and current challenges. Contrast Media Mol Imaging 2017: 7064120

4. Arvind P, Marie-France P, Zaver M (2010) MR molecular imaging of tumor vasculature and vascular targets. Adv Genet 69:1-30

5. Guzmán-de-Villoriaa J, Fernández-Garcíaa P, Mateos-Pérez J, Desco M (2012) Studying cerebral perfusion using magnetic susceptibility techniques: technique and applications. Radiologia 54(3):208-220

6. Sasitorn P, Joana R, Castillo M (2010) Arterial spin labeling in neuroimaging. World J Radiol 2(10):384-398

7. World Medical Association (2013) World Medical Association Declaration of Helsinki: ethical principles for medical research involving human subjects. JAMA 310(20):2191-2194

8. Rafay A, Matthew J, Misun H, Lieberman F, Mountz J (2014) Malignant gliomas: current perspectives in diagnosis, treatment, and early response assessment using advanced quantitative imaging methods. Cancer Manag Res 6:149-170

9. Pope W, Brandal G (2018) Conventional and advanced magnetic resonance imaging in patients with high-grade glioma. Q J Nucl Med Mol Imaging 62(3):239-253

10. Ellingson B, Wen P, Van den Bent M, Cloughesy T (2014) Pros and cons of current brain tumor imaging. Neurooncology 16:2-11

11. Cebeci H, Aydina O, Ozturk-Isikb E, Gumusb C, Inecikli F, Bekard A et al (2014) Assessment of perfusion in glial tumors with arterial spin labeling; comparison with dynamic susceptibility contrast method. Eur J Radiol 83(10):1914-1919

12. Grade M, Hernandez J, Pizzini F, Achten E, Golay X, Smits M (2015) Neuroradiologist's guide to arterial spin labeling MRI in clinical practice. Neuroradiology 57:1181-1202

13. Soni N, Dhanota D, Kumar S, Jaiswal A, Srivastava A (2017) Perfusion MR imaging of enhancing brain tumors: comparison of arterial spin labeling technique with dynamic susceptibility contrast technique. Neurol India 65(5):1046-1052

14. Jiang J, Zhao L, Zhang Y, Zhang S, Yao Y, Qin Y et al (2014) Comparative analysis of arterial spin labeling and dynamic susceptibility contrast perfusion imaging for quantitative perfusion measurements of brain tumors. Int J Clin Exp Pathol 7(6):2790-2799

15. Smits M, Van den Bent M (2017) Imaging correlates of adult glioma genotypes. radiology 284(2):316-331. 
16. Snelling B, Shah A, Buttrick S, Benveniste R (2017) The use of MR perfusion imaging in the evaluation of tumor progression in gliomas. J Korean Neurosurg Soc 60(1):15-20

17. Lev M, Ozsunar Y, Henson J, Rasheed A, Barest G, Harsh G et al (2004) Glial tumor grading and outcome prediction using dynamic spin-echo MR susceptibility mapping compared with conventional contrast-enhanced MR: confounding effect of elevated rCBV of oligodendrogliomas. AJNR 25(2): 214-221

18. Hong M, Zizheng W, Kai X, Zefeng S, Chun Y, Peng X et al (2017) Threedimensional arterial spin labeling imaging and dynamic susceptibility contrast perfusion-weighted imaging value in diagnosing glioma grade prior to surgery. Exp Ther Med 13:2691-2698

\section{Publisher's Note}

Springer Nature remains neutral with regard to jurisdictional claims in published maps and institutional affiliations.

\section{Submit your manuscript to a SpringerOpen ${ }^{\odot}$ journal and benefit from:}

- Convenient online submission

- Rigorous peer review

- Open access: articles freely available online

High visibility within the field

- Retaining the copyright to your article

Submit your next manuscript at $\boldsymbol{\nabla}$ springeropen.com 